\title{
Incidencia de la competencia intercultural en la enseñanza de lengua extranjera, en una institución técnica de Bogotá
}

\section{Incidence of intercultural competition in the teaching offoreign language, in a technical institution of Bogotá}

\section{Diana Cecilia Rodríguez Sanchez ${ }^{1}$}

\author{
Astrid Ramírez Valencia² \\ Universidad Distrital Francisco José de Calda
}

$1 \quad$ Diana Cecilia Rodriguez Sanchez. Licenciatura en Educación Básica con Énfasis en Inglés de la Universidad Distrital Francisco José de Caldas

Correo dicerosanchez@hotmail.com

ORCID: https://orcid.org/0000-0003-0555-9754

\section{$2 \quad$ aramirezv@udistrital.edu.co \\ Teléfono: 3127044844 Universidad Distrital Francisco José de Caldas Bogotá Colombia}

Licenciado en español e inglés. Máster en enseñanza del inglés como lengua extranjera, Ph.D. en Lengua y cultura, formación de profesores de inglés durante más de 25 años. Tiene una larga experiencia como coordinadora, desarrolladora de proyectos, investigadora y gerente en la Universidad Distrital. También tiene varias publicaciones en varias revistas en el área de enseñanza y capacitación. Ha trabajado como investigadora y maestra en la Universidad Distrital Francisco José de Caldas correo aramirezv@udistrital.edu. co ORCID: https://orcid.org/0000-0002-3025-5982

\section{RESUMEN}

En el contexto Bogotano, existen múltiples instituciones dedicadas a la enseñanza del inglés, las cuales en su mayoría han particularizado modos de enseñanza de la lengua, sin embargo, también es muy común encontrarse con aquellas instituciones que optan por organizar sus contenidos alrededor de los temas incluidos dentro de los índices de un libro guía de inglés, dándole énfasis a la enseñanza de la gramática, la sintaxis y el vocabulario, advirtiéndose que esta tendencia no toma en cuenta la incidencia de la competencia socio intercultural en la enseñanza del inglés, como lengua extranjera. 
Los hechos referidos llevaron a analizar al investigador sobre la trascendía de evidenciar si la competencia socio cultural se incluye en las clases de inglés correspondiente a un programa técnico.

Esta investigación acudió a un enfoque cualitativo, con énfasis en la descripción e interpretación de los datos por parte del investigador, con una perspectiva flexible, sistemática y rigurosa, tomando la opinión tanto de los aprendices como del profesor de inglés. Para recoger las muestras de análisis de este estudio se empleó el instrumento de la entrevista aplicada a la población en cuestión.

Los hallazgos sugieren que se contempla la competencia socio intercultural en las clases de inglés, pero no con la debida importancia para contribuir de manera eficaz en la adquisición de la lengua extranjera.

Esta investigación concluye que el aprendizaje de una lengua, en un mundo caracterizado por la globalización, debe partir de la comprensión de los aspectos culturales asociados a la apropiación de una lengua.

PALABRAS CLAVE: Competencia socio cultural, cultura, contexto social, enseñanza de lengua extranjera.

\section{ABSTRACT}

In the Bogotano context, there are multiple institutions dedicated to the teaching of English, which have mostly particularized modes of language teaching, however, it is also very common to meet those institutions that choose to organize its contents around the topics included within the indices of an English guide book, emphasizing the teaching of gramar, syntax and vocabulary, warning that this trend does not take into account the impact of cross-cultural sociocompetitiveness on the teaching of English as a foreign language.
The facts referred to led the researcher to analyze the recentness of demonstrating whether sociocultural competence is included in the English classes corresponding to a technical program.

This research turned to a qualitative approach, with an emphasis on the description and interpretation of data by the researcher, with a flexible, systematic and rigorous perspective, taking the opinion of both the apprentices and the English teacher. The interview tool applied to the population concerned was used to collect the analysis samples from this study.

The findings suggest that socio-intercultural competence in English classes is envisaged, but not with due importance to contribute effectively in the acquisition of the foreign language.

This research concludes that the learning of a language, in a world characterized by globalization, must be based on an understanding of the cultural aspects associated with the appropriation of a language.

\section{KEY WORDS}

Communication skills, foreign language teaching, culture.

\section{INTRODUCCIÓN}

Para comprender la relación entre la cultura y la lengua, es necesario reconocer los elementos inherentes a la comunicación, establecida entre los hablantes de un idioma, al respecto, Cisneros (2008), manifiesta que el lenguaje visibiliza los elementos culturales característicos de una región determinada, es decir que todas las expresiones del lenguaje están cargadas de manifestaciones culturales, costumbres, tradiciones y sin poderse ser ajeno a esta circunstancia. 
Es así como, como Barletta (2009), plantea que la competencia intercultural se asocia con la habilidad de una persona para aplicar sus conocimientos en contextos multiculturales. Esta competencia en el caso de la lengua extranjera, exige el desarrollo de diferentes habilidades, destrezas y actitudes que permiten contextualizar los significados generados para los contextos que se aborden, los cuales, para el caso de la lengua extranjera, podrán ser entendidos solo si se comprende su cultura.

En este sentido, si el profesor de inglés evidencia la relación existente entre la lengua y la cultura cuando enseña este idioma promueve su comprensión lo cual a su vez facilita su aprendizaje. Lo anterior, se ratifica con la definición de Aneas (2015) sobre la competencia intercultural: - "un conjunto de factores que le permiten a una persona comprender las relaciones existentes entre la lengua, el origen geográfico, la identidad, las conductas y los juicios establecidos en contextos determinados."

Con este juicio, se reincide en la importancia de reconocer la cultura como un elemento fundamental en el aprendizaje de una lengua extranjera, porque el contexto de ese idioma es importante para su apropiación, por permitir la interiorización adecuada de un idioma.

Siguiendo las apreciaciones de Di Franco (2005), actualmente es necesario "....aprender una serie de estrategias que derivan de los parámetros culturales en los que se desarrolla una lengua. De hecho, en estos últimos años la relación lengua-cultura se ha convertido en un aspecto fundamental e imprescindible para quien quiere aprender una lengua extranjera" (p. 279).

De esta manera, en la enseñanza de un idioma es fundamental el desarrollo de las competencias interculturales como herramientas fortalecedoras de la adquisición de la lengua.
Por lo tanto, en este artículo se presentan los hallazgos de una investigación relacionada con el uso de situaciones que promueven el desarrollo la competencia intercultural dentro de la cual se establecen vínculos entre la lengua y la cultura, fortalecedor del conocimiento del idioma.

\section{LA COMPETENCIA INTERCULTURAL EN LA ENSEÑANZA DE UNA LENGUA EXTRANJERA}

Existe una relación marcada entre la competencia intercultural y los procesos de aprendizaje de una lengua extranjera, a la cual se le da poca importancia cuando se enseña un idioma, situación que se detecta en el uso de esta lengua. Al respecto, Cardona (2012) explica: _ "El lenguaje (oral, corporal, escrito) no es otra cosa que una herramienta indispensable para la comunicación y por lo tanto para la transmisión de las tradiciones y usos culturales de generación en generación" (p. 113).

Como lo indica este autor, es inevitable dejar de considerar los elementos culturales dentro del diseño de las clases de inglés, por ser elementos fundamentales para apropiarse del idioma; para ello, el profesor debe considerar que los rasgos culturales son esenciales para contextualizar al estudiante con el idioma aprendido.

Es de tener en cuenta, la importancia del profesor, quién deberá en primera instancia ser consciente de sus propios valores culturales, para ser contrastados con aquellos correspondientes a la lengua extranjera que pretende enseñar, esto especialmente por la necesidad de familiarizar las tradiciones de esa cultura que son nuevas para el estudiante, en contraste con aquellos aspectos que le son familiares, y que corresponden a su lengua nativa. 
Como lo explica Sánchez (2012):

"Todo aquel que ha estudiado alguna vez una lengua extranjera conoce que no se trata únicamente de un proceso de aprendizaje de reglas gramaticales, léxico o modelos de conversación. Cuando nos comunicamos... entramos también en contacto con su cultura y nos convertimos en actores de encuentros interculturales (p. 345)."

En este sentido, si el conocimiento sobre una lengua, incluye aquellos aspectos culturales, además de los elementos correspondientes al manejo de la gramática, debería contemplarse también la competencia socio intercultural, por tener un alto grado de importancia en el uso adecuado del idioma, para ratificar lo anterior, Silva (2015), explica que la comprensión de aquellos factores culturales ligados al desarrollo $y$ al uso práctico de una lengua, facilitan y fortalecen la comunicación en esa lengua.

En esta misma dirección, Bravo (2018), coincide con esta perspectiva cuando afirma que: "la lengua es un vehículo de la cultura, en una perfecta simbiosis que en ocasiones ayuda a la actividad docente; Cultura e idioma son, en definitiva, el haz y el envés del aprendizaje de un idioma (p. 3)."

Por consiguiente, se confirma la relación existente entre la cultura y la lengua, de allí la necesidad de contar con un docente que posea una competencia socio intercultural, aplicada a los nuevos enfoques de enseñanza de las lenguas extranjeras, de tal manera que sean considerados los múltiples factores culturales, que afectan la enseñanza de un idioma convirtiéndose en facilitadores de la adquisición del idioma. dos razones que justifican el desarrollo de la competencia intercultural, en la enseñanza de una lengua extranjera. En primer lugar, se debe a la globalización, que ha develado la importancia de generalizar unas normas estándares relacionadas con el inglés, que permitan entablar negocios; como resistencia a esta política, la sociedad se está encaminando al rescate de los valores, manifestaciones y tradiciones locales frente a las globales.

En segundo lugar, La existencia de estudiantes de diversos medios socioculturales que han tenido la experiencia del contacto directo con la lengua, invita a promover enfoques pedagógicos dentro de los cuales se pongan en diálogo, los diversos rasgos culturales correspondientes, tanto a la lengua materna, como a la lengua extranjera, de tal manera que se mantengan en una constante interacción y comunicación que es el fin primordial del dominio de una lengua.

Estas condiciones se dan por circunstancias como la apertura de fronteras, la interconexión entre los habitantes del mundo, la facilidad de acceso al conocimiento mediadas por la Tics, escenarios que obligan a fijar la mirada en el componente cultural dentro del proceso de enseñanza del inglés, como lengua extranjera.

Al respecto conviene mencionar a Martínez (2008), quien explica:

"La institución escolar y el aula misma son, de entrada, espacios de vida, donde, efectivamente, interrelacionan de forma permanente actores sociales, con historias y contextos de vida propios que se encuentran de momento sometidos a procesos de comunicación, conflicto, argumentación, negociación y consenso (p. 288)." 
Esto puede indicar que incluso, el contexto colombiano se caracteriza por la diversidad cultural, a pesar de que se comparta una misma lengua, existen múltiples formas de vivir y de manifestarse, las cuales difieren en los distintos contextos geográficos característicos de nuestro entorno, dejando ver de manera más explícita, la importancia de desarrollar la competencia socio intercultural en los estudiantes.

Por lo tanto, como lo plantea Díaz (2012), en la escuela de hoy el factor cultural desempeña un papel determinante, debido a la relación existente entre las condiciones, historias y percepciones del estudiante, que participa en una actividad de aprendizaje, con sus aptitudes, capacidades y con el contexto situacional, en el cual se desarrolla el aprendizaje de la lengua, se trata entonces reconocer como el contexto permite identificar significados culturales representativos en cada cultura, dependiendo de su interpretación, y de factores tales como el espacio y el tiempo, entre otros.

Por esta razón, el punto clave que los docentes deben tener en cuenta cuando enseñanza una lengua extranjera es considerar desarrollar la competencia socio intercultural en las clases, porque allí se da el significado de las expresiones, los modismos, la prosémica y todos los actos de habla, cuya interpretación dependen de un contexto cultural lo cual permite alcanzar una comunicación en la lengua extranjera, efectiva.

Referida esta situación, se busca desarrollar en los estudiantes una competencia comunicativa, socio intercultural real, dentro de la cual se lidere situaciones, actividades y dinámicas que la ayuden a promover. Como lo explica Paricio (2012): "Todavía hay una atención insuficiente a la dimensión cultural/intercultural en la formación del profesorado" (p. 217).

De acuerdo a esta mirada, se debe considerar esta problemática enfrentada por los docentes de inglés, a la luz de su deber ser como educador, lo que permite sugerir que desde su formación, deberían ser incluidas algunas prácticas de intercambio e inmersión sociocultural con el idioma a enseñar, de tal manera que este profesor este en capacidad de organizar actividades, clubes de lectura, y demás actividades culturales, dentro de las cuales sus estudiantes entren en contacto real, con ella así se llegará a una comunicación fluida $y$ real en esa lengua extranjera.

\section{LA CULTURA Y EL CURRÍCULO DE ENSEÑANZA DEL INGLÉS}

La enseñanza de la lengua extranjera en Colombia ha estado principalmente basada en el desarrollo de las competencias básicas, con el propósito de lograr identificar las estructuras con las cuales sea posible la construcción de textos, el aprendizaje de vocabulario, haciendo énfasis en la competencia gramatical y sintáctica, marginando el uso del idioma extranjero en contextos reales, es aquí donde esta investigación cobra importancia, al sugerir que no se puede desconocer la competencia sociolingüística y cultural, inmersas en la adquisición de una lengua y que son la esencia del aprendizaje contextualizado de un idioma que propicie la comunicación.

Como lo refiere Valencia y otros (2018), respecto a las competencias lingüística y la sociolingüística, en la perspectiva de Chomsky (1970, citado por Tobón, 2013 p. 32), la competencia lingüística es "una estructura mental implícita y genéticamente determinada, que se pone en acción mediante el desempeño comunicativo (uso efectivo de la capacidad lingüística en situaciones específicas)".

Al respecto conviene decir, que esta investigación pudo constatar, que el olvido de la competencia sociolingüística por parte de los docentes, genera dificultades en el dominio de 
la lengua extranjera en sus estudiantes, y de ellos mismos, cuando, teniendo la capacidad lingüística, al tener que usarla adecuadamente en contexto, encuentran impedimentos que los pueden llegar a frustrar cuando necesitan comunicarse en una situación específica.

Lo que interesa entonces, es dar relevancia a la competencia sociolingüística, para llevar al aprendiz a contextualizar la cultura extranjera y llegar a descubrir sus normas, sus tradiciones, sus visiones de mundo, su idiosincrasia inclusive, de esta manera se conseguirá la apropiación de la lengua, cuando el valor de la cultura determine el dominio de la misma.

Lo que se puede apreciar de acuerdo con el análisis de los autores referenciados es que el desarrollo de la competencia intercultural en la enseñanza de una lengua extranjera, tiene gran incidencia y podría potencializar el aprendizaje de la misma, desde la cultura para lograr una apropiación de la misma.

Es un reto para el profesor de lengua extranjera, quien a través de las herramientas Tics, que posibilitan el aprendizaje dan la oportunidad a sus estudiantes para estar en relación con esas otras culturas, a las cuales no hace mucho era casi impensable entrar en contacto, pero que hoy están ahí, solo hay que visibilizarlas y el docente es el posibilitador de esos encuentros, para lograr una comunicación efectiva y con responsabilidad, en ese idioma.

La necesidad de establecer estrategias y dinámicas que permitan contrastar la cultura para practicar las competencias interculturales, se exige de un proceso de renovación constante, en lo que tiene que ver con la metodología y la práctica cotidiana. Para ello, es oportuno, traer las consideraciones de Cisneros (2008), quien resalta el valor de las experiencias costumbres y tradiciones de aquellos elementos vinculados con la cotidianidad de cada idioma, de tal manera que el profesor deberá dar a conocer todos estos elementos inherentes, al dominio de una lengua, lo cual sería posible iniciarlo desde el aula de clase.

Como lo explica Belen (2016), si bien la lengua refleja la cultura, su materialización en las clases de inglés es imperceptible, porque los currículos oficiales hacen mayor énfasis en la competencia gramatical de la lengua, descontextualizada del uso.

Para argumentar esta idea, Areiza y otros (2012), exponen tres razones centrales que reflejan tal dificultad, en cuanto al énfasis de los aspectos culturales inherentes a la enseñanza de la lengua extranjera inglés. Primera, porque los docentes deben cumplir con los objetivos de un currículo escolar, que los llevan a enfatizar en los elementos gramaticales de la lengua, restándole importancia a los aspectos culturales.

Segunda, esto conduce a que el profesor se sienta inhibido y algunas veces atemorizado para emprender otros modos de enseñar que incluyan y enfaticen la relación existente entre la lengua y la cultura, prefiriendo de manera cómoda, enseñar las categorías gramaticales incluidas dentro de currículo, que está obligado a seguir.

Como tercera y último aspecto, señala la resistencia por parte de los estudiantes para aprender elementos culturales, los cuales en algunos casos son completamente ajenos a la realidad de los estudiantes, a sus experiencias, o a sus modos de pensar y como no se explica la razón del aprendizaje de esta cultura, aflorar situación de menosprecio frente al aprendizaje de la cultura extranjera.

De esta manera, se limita la adquisición de la competencia intercultural por parte de 
los aprendices, se agrega además, que en otros casos los elementos culturales giran escasamente alrededor de temas como por ejemplo: las estaciones, las comidas, los nombres de animales y otras temáticas, que aunque hacen parte de las competencias lingüísticas, están descontextualizadas de las competencias interculturales, y en muchos casos, el profesor las presenta de manera ajena a la realidad del estudiante, corriendo el riesgo de generar un rechazo al estar recibiendo un conocimiento que le es completamente ajeno, a su realidad cultural y a su idiosincrasia.

Por estas razones, la situación que generalmente se presenta en las aulas de clase es que los factores culturales solo son vistos superficialmente, considerando solo los logros incluidos en el currículo, desconociéndose la competencia socio intercultural imprescindible para el uso de un idioma con miras a alcanzar la comunicación en el idioma aprendido.

Derakhshan, y otros (2015), sugieren contar con un currículo que presenten unos objetivos claros y unas actividades relacionadas con los elementos socioculturales de la lengua planificadas desde el inicio de un curso, de tal manera que proyecten la cultura a través de las temáticas, proporcionando también el contexto del uso real de la lengua.

Para esto, el fortalecimiento de la competencia intercultural requiere de una planeación previa por parte del profesor, quien debe conocer las relaciones establecidas entre una lengua, su historia y su cultura, la cual está en constante modificación y cambio, de acuerdo con el uso de los hablantes, proceso que debe verse reflejado en las permanentes trasformaciones que el profesor desarrollará en las clases, de tal manera que enfatice los elementos culturales, inherentes a la lengua que está enseñando.
Como se ve en este caso, Almécija (2012), explica que los estudiantes de lengua extranjera deben aprender la cultura, no como un componente desligado de todo el proceso de aprendizaje del idioma, sino como la base central o punto de partida desde la cual se establezcan ejercicios, prácticas y situaciones conversacionales, que ayuden a potenciar desde el inicio sus competencias comunicativas en un contexto cultural real.

Para finalizar, se ratifica esta idea con los planteamientos de Altamar (2015), quien determina que el docente de inglés tiene la responsabilidad de enseñar a los estudiantes la cultura de la lengua que enseña, para que se rompan estereotipos culturales de la lengua que está aprendiendo, que muchas veces son tomados desde temprana edad.

\section{METODOLOGÍA}

Conforme al propósito del presente trabajo de investigación que pretende resaltar la importancia de la competencia socio cultural como eje esencial en el aprendizaje de una lengua extranjera, para lo cual se analizaron algunas estrategias y prácticas que los docentes desarrollan cuando enseñan un idioma; mediante el enfoque metodológico cualitativo, caracterizado, de acuerdo a la perspectiva de Rosario y Castaño (2002), por ser una investigación que produce datos descriptivos, "las propias palabras de las personas, habladas o escritas, y la conducta observable" ( $p$ 7) cuyo énfasis es la descripción e interpretación, por parte del investigador de los datos encontrados u observados.

Ahora bien, para desarrollar este estudio cualitativo, se tuvo en cuenta algunos de los siguientes criterios planteados por Taylor y Bodgan (1986):

Esta investigación es interpretativa, porque una vez se tuvo acceso a las teorías, se procedió a 
recolectar los datos luego fueron interpretados y analizados por el investigador, el cual buscó analizar sus hallazgos, basados en las interpretaciones dadas por las teorías incluidas dentro del marco teórico.

Éste estudio tuvo una perspectiva flexible, porque el fenómeno estudiado se desarrolló tomando en cuenta las distintas perspectivas de los autores incluidos dentro del marco teórico, las cuales sirvieron de referencia para las interpretaciones dadas por el investigador.

Este estudio fue sistemático y riguroso, porque se tuvo en cuenta diferentes aristas y perspectivas científicas, sin embargo, atendiendo a las teorías presentadas por los diferentes autores relacionadas con la temática de la competencia intercultural, y el papel que juega el profesor de inglés en este contexto, el investigador hizo interpretaciones a los hallazgos encontrados dentro de los datos recolectados, en esta investigación.

Finalmente, se pudo establecer que por el objeto de estudio de esta investigación y tomando como referencia la sustentación teórica relacionada con la temática a desarrollar en esta estudio, se puede afirmar que es de tipo descriptivo e interpretativo, especialmente por el papel que juega el investigador, quien pudo dar cuenta de las actividades que pueden ser programadas por el profesor, con las cuales se busca afianzar la competencia intercultural por parte de sus aprendices, de tal manera que el aprendizaje de la lengua extranjera sea significativo.

\section{POBLACIÓN Y MUESTRA}

Esta investigación se llevó a cabo en una institución de carácter privado, donde se imparten programas de formación técnica, que incluyen cuatro cursos de inglés, correspondientes a los niveles: A1, A2, B1, B2, desarrollados durante 18 meses.
Para este estudio se tomaron en cuenta dos cursos, de los cuatro impartidos, correspondientes a los niveles A1 y B1.

La muestra seleccionada fue de 30 participantes en total, cuyas edades oscilaban entre los $17 \mathrm{y}$ 50 años, de los cuales 18 eran mujeres y los restantes eran hombres.

Esta población estaba compuesta por un grupo de 10 profesores de inglés y 15 estudiantes de este idioma

Este estudio se hizo durante casi un año, tiempo durante el cual se desarrollaron los cursos.

\section{INSTRUMENTOS DE RECOLECCIÓN DE DATOS}

Para la recolección de datos se usaron unas encuestas, García (1993) la define como "una técnica que utiliza un conjunto de procedimientos estandarizados de investigación mediante los cuales se recoge y analiza una serie de datos de una muestra de casos representativa de una población o universo más amplio, del que se pretende explorar, describir, predecir y/o explicar una serie de características."

En otras palabras, se trata de utilizar un instrumento que permita obtener una información, que lleven al investigador a establecer consideraciones basadas en los datos arrojados después de la aplicación de este instrumento. Para este caso particular, se indagó si se consideraba relevante la competencia intercultural dentro de las estrategias didácticas usadas por el profesor en la enseñanza del inglés como lengua extranjera.

\section{RESULTADOS E INTERPRETACIÓN}

Los resultados muestran los hallazgos encontrados en la encuesta aplicada tanto a un grupo de profesores, como a un grupo de estudiantes, con el propósito de conocer si era relevante la competencia socio intercultural en 
el desarrollo de las actividades promotoras del aprendizaje de la lengua inglesa, en un grupo de estudiantes de un nivel de inglés en un programa técnico.

La gráfica 1 evidencia el conocimiento de la competencia intercultural tanto en los profesores, como en los estudiantes de un nivel de inglés.

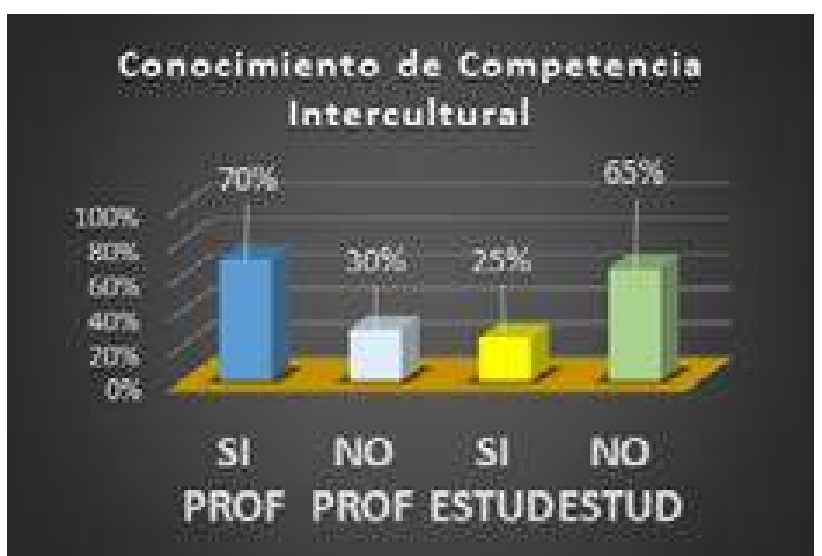

Gráfica 1. Conocimiento de Competencia Intercultural. Fuente: Elaboración propia.

Como se aprecia en la gráfica 1 el $70 \%$ de los profesores encuestados afirmaron conocer el concepto de competencia intercultural, mientras el $30 \%$ lo desconoce, respecto a los estudiantes solamente el $25 \%$ afirman saber la definición del término, mientras el $65 \%$ no. La respuesta de los profesores contradice en parte, las afirmaciones de los estudiantes, pues mientras un alto porcentaje de profesores dice conocer el término, el porcentaje de estudiantes que lo conocen es muy bajo, lo cual indica que los resultados no se presentan de manera proporcional en ambas poblaciones, a pesar de esperarse que, si el profesor conoce esta definición, debería verse reflejado este conocimiento, en las respuestas dadas por los estudiantes.

Como se aprecia en la gráfica 1 el $70 \%$ de los profesores encuestados afirmaron conocer el concepto de competencia intercultural, lo cual indica que es una fortaleza para ellos, lo que se podría dudar es su aplicación en el contexto de la enseñanza de la lengua, en cuanto al $30 \%$ que afirman desconocer esta definición, dando cabida a esta investigación, para fortalecer esta debilidad.

Por otra parte, respecto a los estudiantes solamente el $25 \%$ afirman saber la definición del término, al parecer su docente pocas veces hace mención de este término, sin embargo, aunque es un margen reducido, podría pensarse en la posibilidad que este porcentaje de estudiantes pueden aprender el idioma de forma contextualizada mientras el $65 \%$ no, justificando esta investigación.

Al respecto, Bravo (2018) reconoce la existencia de una relación directa entre la cultura y la lengua, las cuales deberán estar reflejadas en la actividad que hace el profesor, de tal manera que estos ejercicios propicien el manejo de aspectos culturales relacionados con el uso de la lengua en el contexto cultura, para así lograr, el acto comunicativo.

Es aquí donde la visión de Sánchez (2012) cobra importancia al enfatizar la necesidad de incluir elementos culturales dentro de los cursos de inglés, los que por el contrario, tienen la tendencia a incluir mayormente las reglas gramaticales, el vocabulario y en algunas ocasiones la forma de conversación repetitiva, más no las formas de acción comunicativa en contexto, lo cual destaca el rol del profesor, como sujeto decisorio de las actividades de lengua, desarrolladas por los estudiantes, para propiciar una comunicación intercultural en el plan del desarrollo de sus clases.

La segunda pregunta, reflejada en la gráfica 2 , indagó sobre la influencia de la cultura en el dominio de un idioma extranjero. 


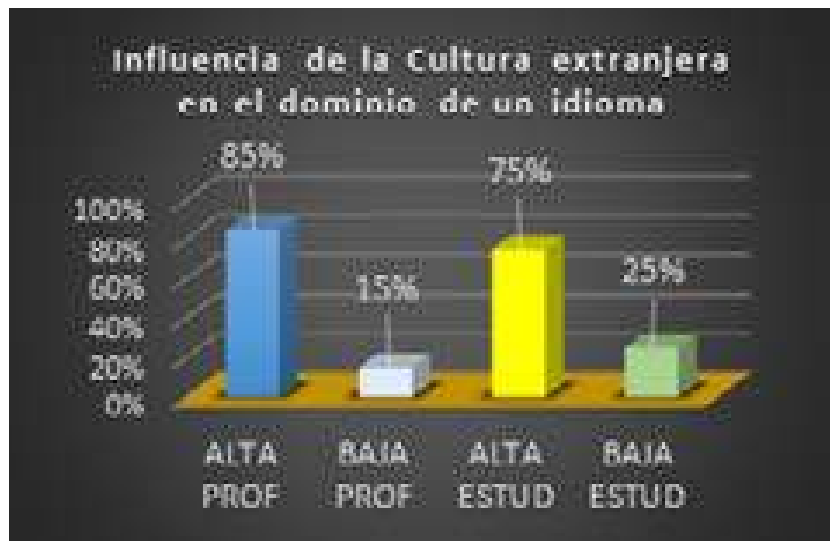

Gráfica 2. Influencia de la Cultura extranjera en el dominio de un idioma.

Fuente: Elaboración propia.

En la gráfica 2 se aprecia una relevancia respecto a la influencia de la cultura extranjera en el conocimiento de la lengua, esto indica que esta investigación tiene un alto grado de importancia acorde con las manifestaciones dadas por la muestra incluida en esta investigación. Sumado a lo anterior, se contempla en esta muestra, que existe una relación directa entre lengua y cultura, sin embargo, se destaca la necesidad de darle mayor importancia y contrastar la cultura de la lengua nativa ( $L 1)$, frente a la lengua extranjera (L2), de tal manera que los elementos culturales correspondientes a la $L 1$, se conviertan en factores facilitadores de la comprensión de los aspectos culturales de la L2.

Claro está, que se debe evitar caer en los estereotipos culturales ofrecidos por los diversos medios de comunicación, los cuales desfavorecen nuestra imagen cultural con respecto a otros países, razón por la cual los docentes deberán convertirse en defensores y promulgadores de nuestros valores culturales, sin desconocer la cultura correspondiente a la lengua que se pretende enseñar.

Con respecto a esta mirada es pertinente mencionar Cisneros (2008), por realzar los aspectos relacionados con la cultura de una lengua usada en el contexto diario del manejo del idioma, de tal manera que este proceso, fortalezca el conocimiento que se tenga sobre la lengua extranjera.

La grafica 3, analiza la frecuencia de inclusión de elementos culturales dentro del currículo.

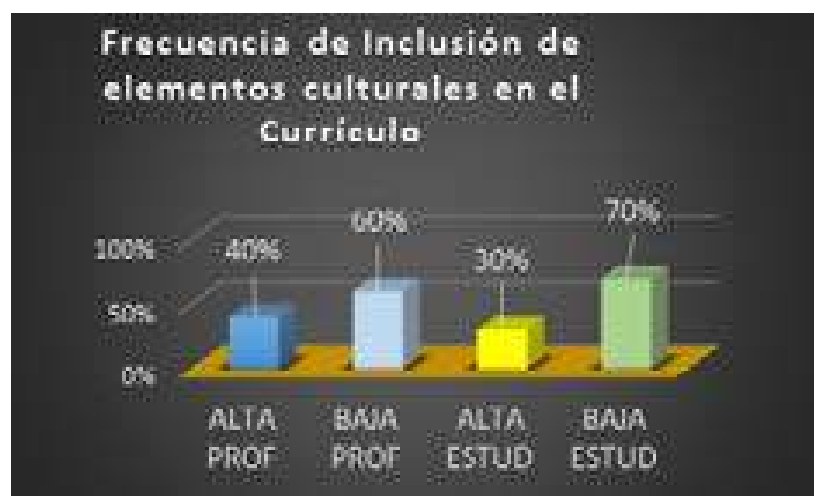

Gráfica 3: Inclusión de elementos culturales en el Currículo. Fuente: Elaboración propia

Respecto al interrogante sobre la frecuencia de inclusión de elementos culturales dentro del currículo, los profesores y estudiantes coinciden en su mirada, los profesores en un $60 \%$, y los estudiantes con un $70 \%$ indican la poca inclusión de elementos culturales en el desarrollo del currículo, lo que da valor a esta investigación, pues indirectamente se reconoce la importancia de ser consciente de incluir y asumir los aspectos correspondientes a la apropiación de un idioma, que llevan finamente a unas dinámicas de comunicación efectivas, esto ocasiona dificultades a los aprendices de una lengua que imposibilita la comprensión de los enunciados en contexto.

Otra de las razones, que subyacen a esta respuesta está motivada por la necesidad de seguir unos contenidos, establecidos por las políticas gubernamentales o por el índice que traen los textos guías para enseñar inglés que deben ser enseñados por el profesor, cuyo propósito busca desarrollar unos contenidos cuya tendencia son gramaticales, más no con templan con profundidad los aspectos culturales de la lengua, ni mucho menos consideran el alcance y la repercusión que ellos tienen dentro 
del proceso de aprendizaje de un idioma para lograr una comunicación real, en contexto.

Lo anterior se puede ratificar con lo determinado por Belen (2016), cuando el docente de inglés debe seguir los currículos de obligatorio cumplimiento, cuyo énfasis está en los aspectos gramaticales, más no culturales de la lengua.

Es necesario recalcar que Areiza y otros (2012), también explican que el docente de inglés esta forzado a cumplir con las estructuras de la lengua, dejando de lado, los elementos culturales del idioma.

Para resolver esta situación, es necesario que el docente abandone su rol de dictador de temas gramaticales, y de cabida a los aspectos culturales de la lengua, convirtiéndolo en dinamizador de actividades, llevando al estudiante a ser sujeto de acción y participación, que lo lleve a exponer, crear y a apropiarse de los elementos culturales de la L2.

Es así como, el profesor necesita diseñar situaciones que reflejen la vida cotidiana, las costumbres, así como de la idiosincrasia correspondiente a la cultura extranjera, propiciando la comunicación con otros, para lograrlo, adaptará los contenidos de acuerdo a las temáticas actuales, de tal forma que despierte el interés y la motivación de quien quiere aprender y dominar la lengua inglesa.

En la gráfica 4 se muestran los resultados asociados con las actividades extracurriculares promovidas por el profesor para que el estudiante conozca la cultura correspondiente a la lengua extranjera.

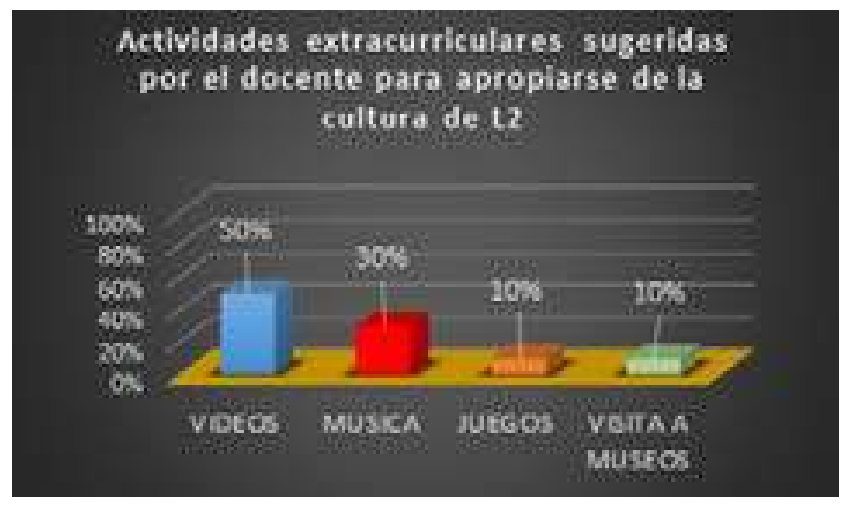

Gráfica 4: Actividades extracurriculares promotoras de la cultura de L2. Fuente:

Elaboración propia

En estos resultados se aprecia el vídeo como actividad con un $50 \%$ de uso por el profesor, para dar a conocer los aspectos culturales de la lengua, por ser un recurso de gran aceptación entre los estudiantes, con el cual se consigue al mismo tiempo incrementar su interés por el reconocimiento de las características culturales de un idioma.

De igual manera, se espera que esta ayuda audiovisual contribuya a la eliminación de estereotipos por parte del estudiante, abriendo puertas a la aceptación de la diversidad cultural, valorando al mismo tiempo, los artefactos culturales de su lengua nativa.

Sin embargo, es claro que no basta únicamente son sugerir ver el video con propósito cultural, es necesario que el profesor acuda a otros recursos didácticos que lleven al estudiante a abstraer los elementos culturas de la lengua implícitos en este tipo de ayudas, de tal manera que el estudiante interiorice, valore y aprecie su cultura en contraste con las detectadas en el video, para convertirse en una fuente de conocimiento cultural dela lengua extranjera.

Otro recurso al que el profesor puede acudir corresponde a la música, que en la gráfica contó con un $30 \%$ de aceptación, ocupando 
el segundo recurso sugerido por el docente, lo cual nos señala que esta consideración deja pendiente la contextualización cultural, para identificar, por ejemplo, su origen, la fuente, o el contexto histórico, cuyo análisis puede ser usado como tema para una investigación futura.

Habría que decir también que el profesor tiene el compromiso de ser facilitador del contacto con la cultura y la utilización de recursos tales como la música para este fin, de tal forma que incluso el estudiante puede entrar en diálogo con los elementos culturales correspondientes a la lengua extranjera y así llevarlo a profundizar en esta temática de manera autónoma y efectiva, a lo largo de su proceso de aprendizaje de lengua.

En último lugar, se sugiere el uso de juegos y las visitas a museos, las cuales demuestran poca aceptación, seguramente por el bajo conocimiento que los estudiantes tienen sobre este tipo de actividades, además por su poca familiaridad, a pesar de la gran posibilidad que ofrecen estos recursos para adquirir con mayor facilidad los elementos culturales, correspondientes a las comunidades extranjeras.

Esta investigación insiste en la perspectiva de Cisneros (2008), quien considera valiosa la inclusión de costumbres y tradiciones relacionadas con la vida cotidiana, correspondiente a las comunidades extranjeras, de tal manera que el profesor tenga en cuenta estos aspectos dentro de sus sesiones de clase, para familiarizar al estudiante con ellas, dándoles la importancia que se merecen dentro del proceso de aprendizaje de la lengua; de esta forma se inquietará al estudiante para acceder a estos elementos culturales, que son parte indispensable en la apropiación de un idioma extranjero.

Pongamos la perspectiva de Almécija (2012) quien explica que los estudiantes necesitan aprender la cultura, convirtiéndola como eje central cuando el profesor decida escoger ejercicios y situaciones que la promuevan, para esta investigación se evidenció que la actividad con mayor reconocimiento y como una fuerte fuente cultural son los vídeos, especialmente por mostrar situaciones de contexto reales, con las cuales los estudiantes tienen la oportunidad de entrar en contacto y extraer aquellos elementos fortalecedores de su aprendizaje de la lengua extranjera, pero que lamentablemente solo son usados esporádica y superficialmente, sin ahondar en los detalles previamente explicados.

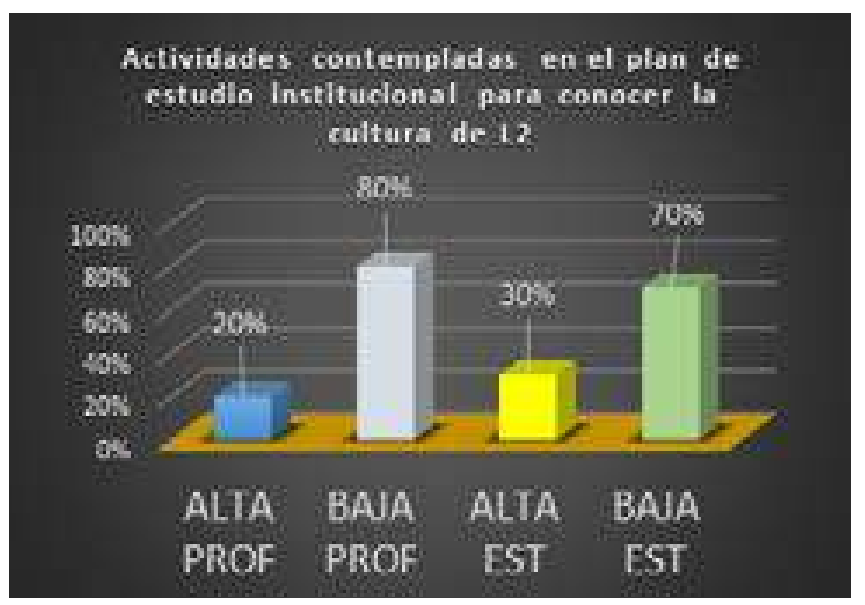

Gráfica 5: Actividades contempladas en el plan de estudio institucional para conocer la cultura de L2. Fuente: Elaboración propia

En la gráfica 5, se aprecia que los profesores con un $80 \%$ y los estudiantes con un $70 \%$ coinciden en señalar las escasas actividades sugeridas contempladas por la institución para promover el conocimiento de la cultura extranjera, en este caso a la lengua inglesa, de tal manera que la oportunidad de entrar en contacto con las costumbres y la idiosincrasia de las personas que usan esta lengua es mínima.

Esta situación seguramente se debe a las pocas oportunidades que tienen los profesores y los estudiantes para por ejemplo viajar a países de habla inglesa, dentro de los cuales puedan entrar en contacto directo con las costumbres 
de esas comunidades, seguramente por razones económicas; seguramente, tampoco se programan actividades extra curriculares, dentro de las cuales los estudiantes pueden entrar en contacto con otras personas que hablen inglés ni tampoco probablemente están propiciando encuentro asincrónicos con instituciones extranjera donde hallan estudiantes extranjeros, de tal forma que se den encuentros virtuales, colaborativos con otras instituciones que también enseñen idiomas, estos encuentros también podrían darse dentro de nuestro contexto colombiano, a nivel inter institucional, dando muestra de un trabajo colaborativo.

En algunos casos, la imposibilidad de emprender estas acciones se atribuyen a múltiples factores como; el tiempo, la logística, y ante todo los costos que implican la planeación y el desarrollo para llevar a cabo de este tipo de actividades, las cuales deberán seguramente ser asumidos por los estudiantes, habría que preguntarles, si la institución está dispuesta a asumir este reto, con el propósito de tener la oportunidad de entrar en contacto directo con los elementos culturales correspondientes a esta lengua, así sea dentro de nuestro contexto bogotano, allí se deben programar variedad actividades, donde el estudiante tiene la oportunidad de identificar múltiples elementos culturales, correspondientes a la lengua extranjera.

Con el pasar del tiempo, estas acciones tendrán sus efectos en la calidad de los cursos ofrecidos incidiendo al mismo tiempo en la demanda de los mismos, lo que redundará en el beneficio de todos los integrantes de la comunidad académica.

\section{CONCLUSIONES}

En este escrito se evidenció la relación existente entre la lengua y la cultura, por lo tanto, el profesor de inglés debe resaltar la importancia de esta dependencia, tomándola como eje fundamental dentro de la planeación de un curso de inglés.

Con esto se comprende que, el profesor de inglés necesita ir más allá de la explicación gramatical y de la familiarización con el vocabulario a la cual comúnmente se atiende dentro de las clases de inglés, para considerar la inclusión de los elementos culturales vitales para afianzar el conocimiento de la lengua y la importancia del uso en la intención de comunicar en contexto.

A través de este escrito se pudo determinar que existe una tendencia por priorizar los elementos lingüísticos correspondientes a la lengua, más no a los factores culturales, esto debido a la necesidad de desarrollar un currículo lleno de contenidos gramaticales, como lo contemplan las políticas educativas, descontextualizando la lengua de su entorno cultural, determinante del significado.

Esta revisión llevó a percibir que los docentes formadores de lengua extranjera, si trabajan distintos de materiales para el aprendizaje de la misma, pero su debilidad se detecta es en la falta de contextualización de los mismos, con la competencia intercultural de la comunidad inglesa, en este caso específico.

El anterior hallazgo podría resolverse si el profesor de inglés organiza y escoge múltiples actividades dentro de las cuales el estudiante tendrá la oportunidad de entrar en contacto con la cultura, correspondiente a la lengua extranjera, escudriñando minuciosamente todas sus variables culturales.

De esto podemos extraer que el profesor de inglés cuando organiza múltiples actividades, donde los estudiantes entren en contacto con la realidad cultural de la lengua extranjera aprendida, se podrá propiciar una necesidad de 
uso de la lengua por parte de los aprendices, logrando una comunicación eficaz, con miras a fomentar después de un tiempo, un pensamiento crítico y reflexivo, frente a los elementos culturales aprendidos, que indirectamente lo llevarán a afianzar sus valores culturales.

Este estudio propone que para lograr lo anteriormente planteado, el profesor de inglés al planear actividades dentro y fuera del aula de clase, requiere el apoyo institucional, con las cuales sea posible que el estudiante se exponga a diferentes situaciones culturales relacionadas con la lengua extranjera que está adquiriendo, como por ejemplo; visitas guiadas por hablantes de inglés dentro o fuera de la ciudad, eventos sobre la cultura de ese país, homenajes a escritores de habla inglesa, así se logrará dar un aprendizaje más significativo para el aprendiz, con la intencionalidad de comunicarse eficazmente.

Para terminar, se considera indispensable que las instituciones donde se aprende la lengua extranjera, faciliten tanto a los profesores, como a los estudiantes, el contacto directo con esa lengua nativa, a través de viajes programados o con encuentros virtuales con hablantes de inglés donde se fortalezcan los valores culturales y se evidencie el uso en contexto de la lengua extranjera, que será una experiencia exitosa para quien aprende y para la institución tendrá un reconocimiento por apoyar este tipo de iniciativas que redundarían en prestigio académico traducido posteriormente en el aumento del número de aspirantes de ingreso a una institución, que ofrezca esta novedosa posibilidad. 


\section{ANEXO}

Encuesta de percepción

La siguiente encuesta será aplicada a 15 estudiantes del nivel A1 del Programa de Conocimientos Académicos en Inglés de la Corporación Iberoamericana de Estudios-CIES, y a 15 docentes de inglés que busca identificar la relación existente entre la competencia socio intercultural y la enseñanza del inglés.

\section{Encuesta sobre competencias interculturales en la enseñanza de una Lengua Extranjera}

La presente encuesta tiene una finalidad investigativa y su objetivo no es de carácter evaluativo, por lo cual se le pide responder con total sinceridad y tomarse el tiempo y espacio necesarios para dejar claras sus ideas.

\section{Competencias interculturales}

1. ¿Sabe lo que es "competencia intercultural"? ¿En algún momento ha sido mencionada en sus clases?

2. ¿De 1 a 10, con cuánto calificaría el conocimiento que tiene sobre la cultura de los países de habla inglesa? Mencione algunos rasgos representativos.

3. ¿Considera necesario conocer sobre la cultura de los países de habla inglesa en el proceso de comprensión de dicho idioma?

4. Además de los conocimientos lingüísticos, ¿qué otras habilidades, actividades o actitudes considera necesarias para comprender de manera integral el idioma inglés? (Hablar con nativos, ver noticias de esos países, asistir a eventos, etc.) ¿Por qué? 


\section{Aprendizaje significativo}

5. ¿Ha conocido o interactuado con personas de habla inglesa nativas? ¿Cómo fue la experiencia?

6. ¿Acostumbra a consumir contenido en inglés producido en países de habla inglesa? (noticieros, libros, películas, redes sociales, música, series, etc.)

7. ¿Conoce museos, galerías o teatros en los cuales se exponga el arte y la cultura de los países de habla inglesa' ¿Ha asistido a ellos?

8. ¿Considera importante realizar este tipo de interacciones, consumir estos productos y asistir a este tipo de lugares en el proceso de aprendizaje de inglés? ¿Por qué?

9. ¿Podría mencionar algunas pautas de comportamiento propias de los habitantes de países de habla inglesa? Por ejemplo, la manera de iniciar un saludo o la forma de ordenar en un restaurante y ¿podría compararlas con la forma como dichas pautas se evidencian aquí?

\section{Estrategias didácticas}

10. ¿Qué herramientas, además de las clases magistrales, emplean los docentes para el aprendizaje del idioma?

11. ¿La Corporación y los docentes promueven la participado en eventos culturales o actividades conversacionales con personas nativas para mejorar el proceso de aprendizaje del idioma?

12. ¿Las clases incorporan elementos culturales para mejorar el aprendizaje? ¿Cuáles?

13. ¿Qué herramientas incorporaría usted a las clases para mejorar su conocimiento sobre los países de habla inglesa?

Agradecemos su participación y sinceridad. 


\section{REFERENCIAS BIBLIOGRÁFICAS}

Almécija, I. (2012). "El cine en el aula de inglés como transmisor de cultura". Máster profesorado Secundaria. Universidad de Almería.

Altamar, W. (2015). La cultura como herramienta para la enseñanza del español como lengua extranjera. Red académica de español como lengua extranjera - EnRedELE - agosto 18, 19 y 20 de 2015.

Aneas, M. (2015). Competencia intercultural, concepto, efectos e implicaciones en el ejercicio de la ciudadanía. Facultad de Pedagogía, Universidad de Barcelona, España

Areiza, R., Cisneros, M., y Tabares. L. (2012). Sociolingüística: enfoque pragmático y variacionista. Bogotá: ECOE.

Barletta, N. (2009). Intercultural Competence: another challenge. PROFILE Issues in Teachers' Professional Development, $11,143-158$

Belén, A. (2016). La competencia intercultural y el papel del profesor de lenguas extranjeras. Centro Virtual Cervantes, 12(2), 493-504.

Bravo, E. (2018). La competencia cultural: desarrollo y límites en la docencia. Revista Electrónica del Lenguaje, 5(2), 12-66.

Cardona, L. (2012). Relaciones culturales en el aprendizaje de lengua extranjera. Sophia, 8(2), 112-119.
Cisneros, M. (2008). “Educación, lenguaje y poder desde una mirada sociolingüística". En: Primer Congreso Internacional de Sociolingüística, Bahía Blanca (Argentina), 28 al 30 de nov. de 2007.

Derakhshan, A., Tamaskani, R., y Faribi, M. (2015). Issues in language learning strategies. International Journal of Social Sciences and Education. 5(4), 187-199.

Di Franco, S. (2005). La relación lengua-cultura en el aprendizaje de E/LE por parte de los italianófonos. ASELE, 16(2). 279302.

Díaz, A. (2012). La formación cultural: una propuesta sobre cómo asumirla y estudiarla. Ikala Revista de Lenguaje y Cultura 17(3), 223-229.

Diaz, A., \& Quiroz, R. (2012). ¿Cómo ven la formación cultural los estudiantes de la Licenciatura en Lenguas Extranjeras?, Lenguaje, 40(1), (pp. 17-40).

Garcia M, Ibañez J, Alvira F. (1993) El análisis de la realidad social. Métodos y técnicas de Investigación. Madrid: Alianza Universidad Textos. p. 141-70

Gonzáles, H. Ramírez, A, Salazar, P. (2018) Las Tic en el mejoramiento de las competencias en lenguas extranjeras de los profesores de inglés. Editorial Eduard Javier Ordoñez - Cali: Universidad Santiago de Cali.

Martínez, B. (2008). El aprendizaje de la cultura y la cultura de aprender. UAEMex, 48, 287-307. 
Paricio, M. (2012). Competencia intercultural en la enseñanza de lenguas extranjeras. Porta Linguarum 21(4), 215-226.

Rosario y Castaño (2002). Introducción a la metodología de investigación cualitativa. Revista de Psicodidáctica

Sánchez, M. (2012). El desarrollo de la competencia intercultural en la clase de ELE. Universidad de Economía de Bratislava.

Silva, D. (2015). Innovación en la práctica docente. Revista Vinculando, 4(2), 2259.

Taylor, S. y R.C. Bogdan (1986). Introducción a los métodos cualitativos de investigación. Paidós, Barcelona. 